A moving-coil designed micro-mechanics tester with application on MEMS

This article has been downloaded from IOPscience. Please scroll down to see the full text article.

2007 Meas. Sci. Technol. 183612

(http://iopscience.iop.org/0957-0233/18/11/046)

View the table of contents for this issue, or go to the journal homepage for more

Download details:

IP Address: 159.226.231.78

The article was downloaded on 12/06/2010 at 07:48

Please note that terms and conditions apply. 


\title{
A moving-coil designed micro-mechanics tester with application on MEMS
}

\author{
Yong Huan, Taihua Zhang and Yemin Yang \\ State Key Laboratory of Nonlinear Mechanics (LNM), Institute of Mechanics, \\ Chinese Academy of Sciences, Beijing 100080, People's Republic of China \\ E-mail: zhangth@1nm.imech.ac.cn
}

Received 4 April 2007, in final form 17 July 2007

Published 16 October 2007

Online at stacks.iop.org/MST/18/3612

\begin{abstract}
A moving-coil designed micro-mechanics tester, named as MicroUTM (universal testing machine), is in-house developed in this paper for micro-mechanics tests. The main component is a moving coil suspended in a uniform magnetic field through a set of springs. When a current passes through the coil, the electromagnetic force is proportional to the magnitude of the current, so the load can easily be measured by the current. The displacement is measured using a capacitive sensor. The load is calibrated using a Sartorius BP211D analytical balance, with a resolution/range of $0.01 \mathrm{mg} / 80 \mathrm{~g}$ or $0.1 \mathrm{mg} / 210 \mathrm{~g}$. The displacement is calibrated using a HEIDENHAIN CT-6002 length gauge with an accuracy of $\pm 0.1 \mu \mathrm{m}$. The calibration results show that the load range is $\pm 1 \mathrm{~N}$ and the displacement range is $\pm 300 \mu \mathrm{m}$. The noise levels of the load and displacement are $50 \mu \mathrm{N}$ and $150 \mathrm{~nm}$, respectively. The nonlinearity of the load is only $0.2 \%$. Several in-plane load tests of the MEMS micro-cantilever are performed using this tester. Experimental results, with excellent repeatability, demonstrate the reliability of the load measurement as well as the flexible function of this tester.
\end{abstract}

Keywords: micro-mechanics, tester, design, MEMS

(Some figures in this article are in colour only in the electronic version)

\section{Introduction}

The micro-mechanics test technique is significant for the mechanical theory research and engineering application. Progress in this area of mechanics is seriously hampered by the lack of suitable instrumentation and methods, e.g. design and manufacturing of controllable mechanical loading fixtures for small-scale samples [1]. Tension, compression and bending tests are favorites because the mechanical model is simple and the results have definite physical meanings. Samples of the size of a few millimetres and larger can be tested using conventional universal testing machines [2-4], which usually have a motor as the actuator and a strain gauged load cell as the load measuring means. However, for micro-sized samples such as micro-structures in MEMS (microelectromechanical system) and micro-fibers, these testers are not suitable due to the load and displacement resolution. Some alternative means need to be developed [5].
One kind of device adopts piezoelectric stacks as the actuator in order to improve the displacement resolution [6-8]. However, it is difficult to entirely eliminate the creep, lag and nonlinearity of the piezoelectric stack. Furthermore, these devices still use the strain gauged load cell, which confines the load resolution. Hence Read adopted two parallel spring-steel strips as a force sensor to replace the strain gauged load cell $[9,10]$. Through measuring the deformation of the spring by the eddy-current displacement sensor, the force can be recorded as the product of the deformation and the stiffness of the spring. This method can greatly improve the load resolution as long as the spring is flexible enough. However, the stability of the loading orientation will be influenced by the low stiffness spring.

Another kind of device is designed on the basis of electromagnetism, getting rid of the load cell $[11,12]$. These devices have two moving magnets suspended at the orifice of the fixed coil, shown in figure 1(a). The electromagnetic force 


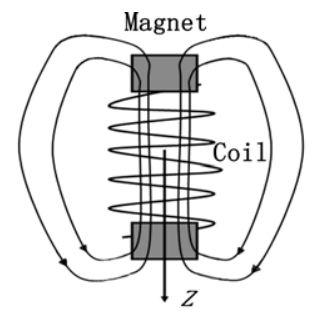

(a)

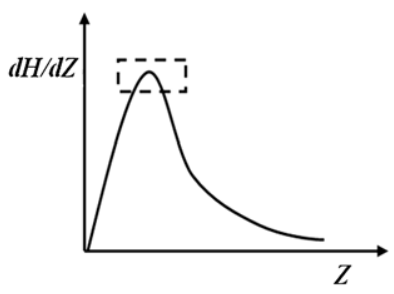

(b)
Figure 1. Schematic illustration of the function conditions of the moving-magnet designed devices: (a) two moving magnets are located at the orifices of the immovable coil, $(b)$ the relationship between the magnetic field gradient $\mathrm{d} H / \mathrm{d} Z$ derived by the coil and the magnet position $Z$. The moving-magnet designed devices function in the vertex segment of the magnetic field gradient (marked with a dashed box).

$F_{\mathrm{e}}(t)$ is proportional to the magnetic field gradient $\mathrm{d} H / \mathrm{d} Z$, which is further proportional to the coil excitation current $I(t)$, i.e. $F_{\mathrm{e}}(t) \sim \mathrm{d} H / \mathrm{d} Z \sim I(t)$. Therefore, the load can easily be measured by the current. These devices function in the vertex segment of the magnetic field gradient in order to get the high sensitivity of the load, marked with a dashed box in figure $1(b)$. However, the relationship $F_{\mathrm{e}}(t) \sim \mathrm{d} H / \mathrm{d} Z \sim I(t)$ requires that the magnetic field gradient be constant throughout the whole displacement range. This is difficult on technology. In fact, the magnetic field gradient $\mathrm{d} H / \mathrm{d} Z$ changes very quickly at the orifice of the coil. Therefore, the whole displacement range has to be strictly limited in order to keep the good linear relationship $F_{\mathrm{e}}(t) \sim \mathrm{d} H / \mathrm{d} Z \sim I(t)$.

In order to improve the weakness mentioned above, a moving-coil designed tester, named as MicroUTM (universal testing machine), is developed in this paper.

\section{Design of the tester}

A testing machine usually contains an actuator and the means of measuring load and displacement. The present MicroUTM has satisfied this condition by having a coil suspended in a magnetic field acting as the actuator as well as the load measuring means, a capacitive sensor as the displacement measuring means. The dimensions of the coil, the magnet

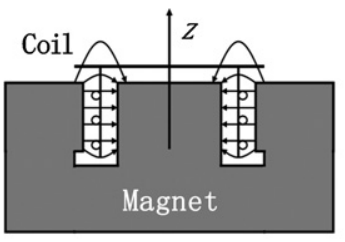

(a)

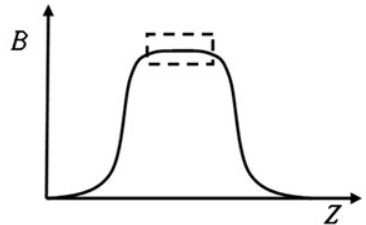

(b)
Figure 3. Schematic illustration of the function conditions of the present MicroUTM: $(a)$ a coil is located at the orifice of the immovable magnet, $(b)$ the relationship between the magnetic density $B$ derived by the magnet and the coil position $Z$. The present MicroUTM functions in the uniform segment of the magnetic field (marked with a dashed box).

and the displacement sensor are about $35 \mathrm{~mm} \times \varnothing 35 \mathrm{~mm}$, $60 \mathrm{~mm} \times \varnothing 60 \mathrm{~mm}$ and $40 \mathrm{~mm} \times \varnothing 20 \mathrm{~mm}$, respectively.

The geometry of the device is cubic, as shown in figure 2(a). The dimension of the present MicroUTM is about $400 \mathrm{~mm} \times 270 \mathrm{~mm} \times 180 \mathrm{~mm}$. The structure is illustrated in figure $2(b)$. The displacement sensor, the coil and the magnet are enwrapped in the body. The upper grip is mounted on the three-dimensional stage so that the sample position can be adjusted. The lower grip is mounted on the load axis. The tested sample is fastened between the upper and lower grips.

The profiles of the coil and the magnet are illustrated in figure 3(a). The fixed magnet supplies an approximately uniform magnet field. When a current passes through the coil, the electromagnetic force $F_{\mathrm{e}}(t)$ is produced and the coil moves up and down to load the sample. If the magnetic density $B$ is constant, the electromagnetic force $F_{\mathrm{e}}(t)$ is proportional to the excitation current $I(t)$, i.e. $F_{\mathrm{e}}(t) \sim I(t)$. Therefore, the load can easily be measured by the current. It is notable that the linear relationship $F_{\mathrm{e}}(t) \sim I(t)$ can only be satisfied in the uniform segment of the magnetic field, marked with a dashed box in figure 3(b). A uniform and deep magnetic field can be easily achieved with technology, so good load linearity as well as a relatively long displacement range is predicted.

\section{The mechanical model}

In micromechanics tests, even a tiny friction can badly influence the load measurement. Hence, the displacement

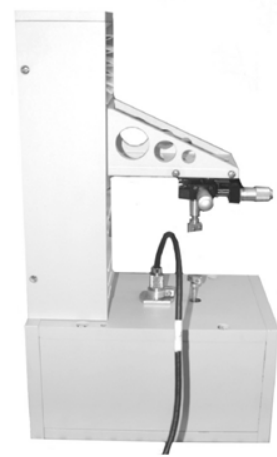

(a)

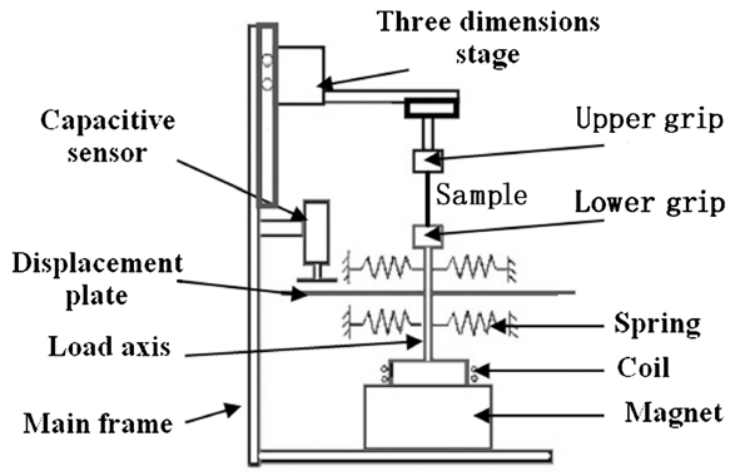

(b)

Figure 2. Schematic diagram of the structure of the MicroUTM. It has a moving coil suspended in a uniform magnetic field as the actuator as well as the load measuring means, and a capacitive sensor as the displacement measuring means. 


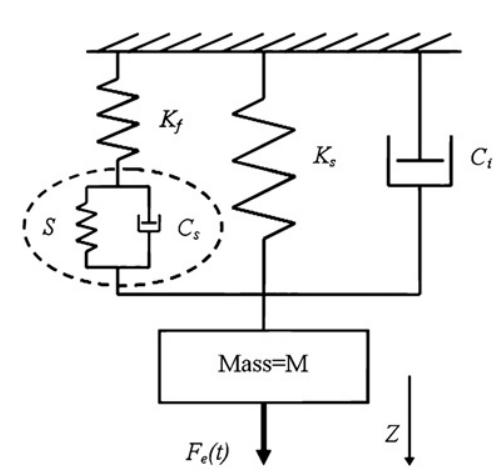

Figure 4. A mechanical model of the MicroUTM. In static tests, all damping can be ignored. The stiffness of the frame and the spring can influence the load and displacement measurements on the sample.

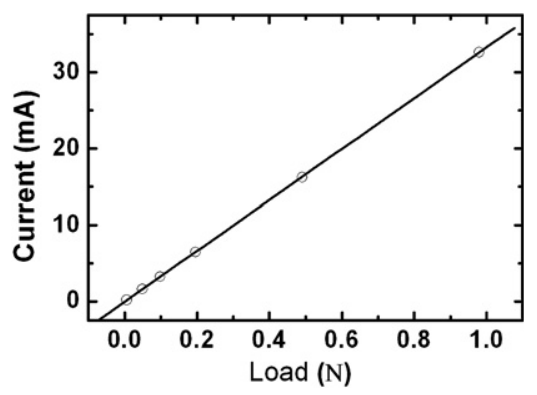

Figure 5. Relationship between the excitation current and electromagnetic force. The nonlinearity is only $0.2 \%$.

is measured using a capacitive sensor, which is a non-contact measuring technique. Furthermore, a set of springs is used to support the moving mass and to orient the movement of the load axis. However, new problems are introduced, that is, the responses of the device and the sample are coupled together, and the electromagnetic force does not just represent the load on the sample. The mechanics model of the MicroUTM is illustrated in figure 4 , where
$M \quad$ is the moving mass, including the mass of the coil, the load axis, the displacement plate and the lower grip;

$C_{\mathrm{i}} \quad$ is the damping of the moving parts, which can be ignored in the static tests;

$K_{\mathrm{f}} \quad$ is the stiffness of the machine frame, which results from the deformation of the main frame, the threedimensional stage and the upper grip;

$K_{\mathrm{S}} \quad$ is the stiffness of the spring in the vertical direction;

$S \quad$ is the stiffness of the sample, which is exactly what need to be measured;

$C_{\mathrm{s}} \quad$ is the damping of the sample, which can also be ignored in the static tests.

In the static tests, all damping can be ignored. The test system complies with the following equations:

$$
\left\{\begin{array}{l}
K Z=F_{\mathrm{e}}(t) \\
F_{\mathrm{e}}(t)=B l I(t) \\
K=\left(S^{-1}+K_{\mathrm{f}}^{-1}\right)^{-1}+K_{\mathrm{s}}
\end{array}\right.
$$

where $Z$ is the displacement in the vertical direction, and $K$ is the equivalent stiffness of the test system. This means that the load on the sample is recorded as the difference between the electromagnetic force and the spring force induced by the displacement.

\section{Calibration work}

A series of calibration work was performed in order to investigate the performance of the MicroUTM, including the load, the displacement and the spring calibration.

The purpose of the load calibration is to derive a relationship between the load and the excitation current. A Sartorius BP211D analytical balance, with a resolution/range of $0.01 \mathrm{mg} / 80 \mathrm{~g}$ or $0.1 \mathrm{mg} / 210 \mathrm{~g}$, was used to perform the calibration. Firstly, the coil was suspended in the middle of the whole range. The current and the position were denoted as $I_{1}$ and $z_{0}$, respectively. Secondly, a weight was kept on the load axis. The coil went down. Thirdly, the current was increased

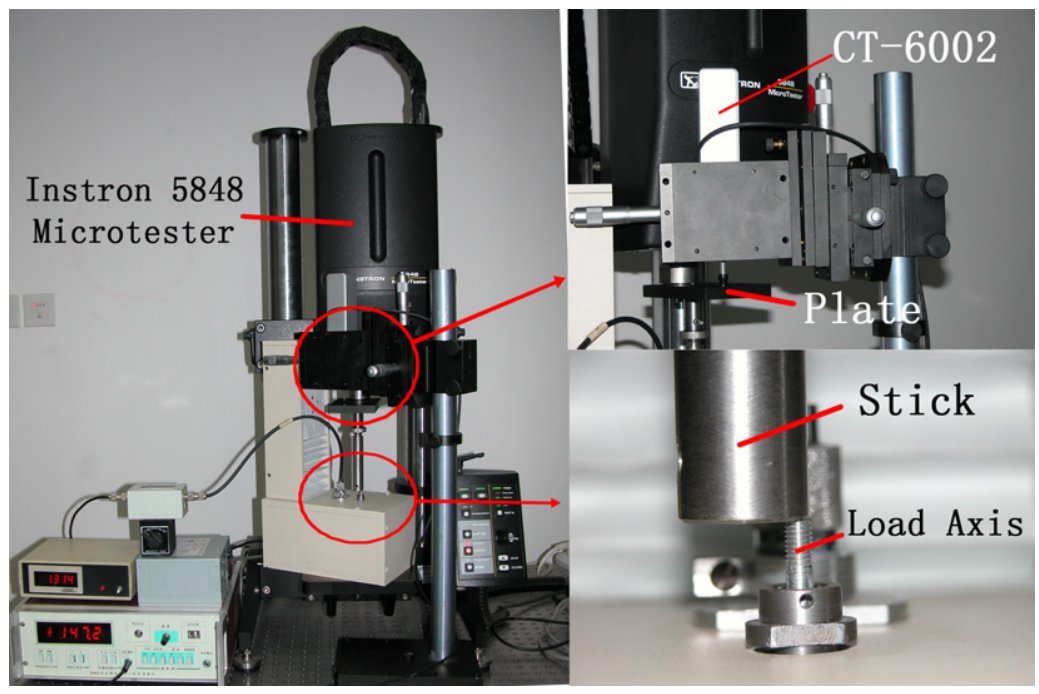

Figure 6. Displacement calibration process. An Instron 5848 Microtester moved the probe of the CT-6002 length gauge and the load axis of the present MicroUTM together. The output voltage of the displacement sensor in the MicroUTM and the reading of the CT-6002 length gauge (acting as the criterion) were recorded together. 


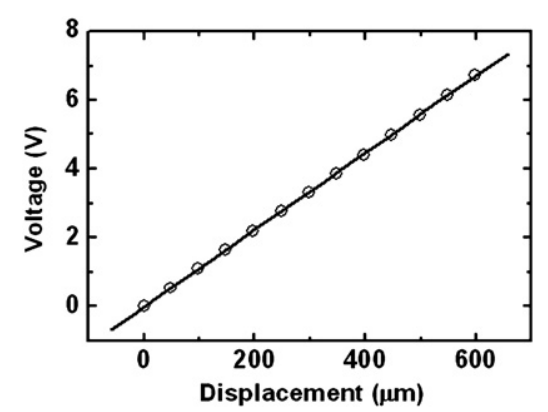

Figure 7. Relationship between the output voltage of the capacitive sensor and the displacement reading of the CT-6002 length gauge. The nonlinearity is only $0.4 \%$.

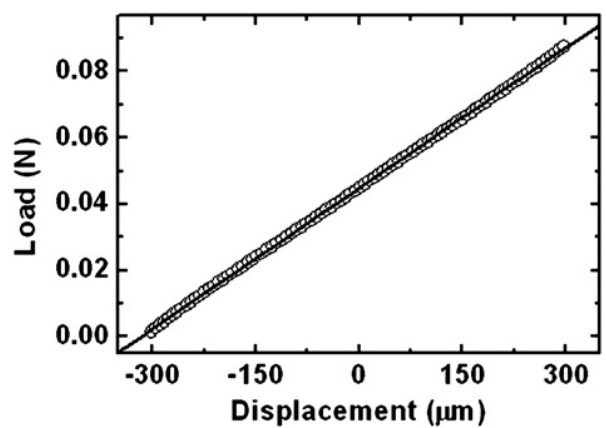

Figure 8. Load-displacement curve of the spring. The average stiffness is $140 \mathrm{~N} \mathrm{~m}^{-1}$ and the nonlinearity is $0.8 \%$ throughout the $\pm 300 \mu \mathrm{m}$ travel.

to $I_{2}$ to make the coil return to the original position $z_{0}$. The electromagnetic force derived from the current difference $\Delta I=I_{2}-I_{1}$ balanced the added weight, which was measured by the BP211D analytical balance. A total of six different weights were used in the calibration work. The relationship between the excitation current and the load is shown in figure 5. The nonlinearity of the load is only $0.2 \%$. The load range is $\pm 1 \mathrm{~N}$, which is confined by the current capacity of the test system.

The purpose of the displacement calibration is to obtain the sensitivity and the linearity of an untapped sensor, namely the capacitive sensor. The HEIDENHAIN CT-6002 length gauge with accuracy of $\pm 0.1 \mu \mathrm{m}$ was used to perform the calibration. The probe of the CT-6002 length gauge was settled on a plate fixed on the load axis of an Instron 5848
Microtester, which only acted as an actuator. At the same time, the load axis of the present MicroUTM was pushed by a steel stick mounted on the plate; thus, the probe of the CT6002 length gauge and the load axis of the present MicroUTM moved together, shown in figure 6 . The relationship between the output voltage of the displacement sensor in the present MicroUTM and the reading of the CT-6002 length gauge is shown in figure 7 . The displacement range is $\pm 300 \mu \mathrm{m}$ and the nonlinearity is $0.4 \%$. It is notable that the displacement range is limited by the linearity of the displacement sensor instead of the uniformity of the magnetic field.

The noise level of a testing machine is the signal fluctuation even without any external load or displacement. In practice, the noise level determines the ability of the testing machine to measure the minimum load and displacement. In order to inspect the noise level of the present MicroUTM, the coil was suspended somewhere by a constant current. The fluctuating load signal, representing the load noise level, was recorded as $50 \mu \mathrm{N}$. In the same way, the fluctuating displacement signal, representing the displacement noise level, was recorded as $150 \mathrm{~nm}$.

Since the load on the sample is recorded as the difference between the electromagnetic force and the spring force induced by the displacement, the performance of the spring is significant for the load measurement. The load-displacement curve of the spring can be obtained by moving the coil up without any external load on the load axis, shown in figure 8 . The average stiffness in the vertical direction is $140 \mathrm{~N} \mathrm{~m}^{-1}$ throughout $\pm 300 \mu \mathrm{m}$ range. The nonlinearity of the load is $0.8 \%$.

\section{The application experiment}

The micro-cantilever bonded onto a glass substrate is a typical structure extensively used in sensors, actuators, RF-switches etc. In a lot of MEMS devices, especially in electrostatic actuators $[13,14]$, the cantilever endures a tiny load parallel to the substrate, meaning that an in-plane load test is needed. Unfortunately, former researches were only concerned with the out-of-plane load tests [15-19], in which the stress condition at the bonding interface is relatively simple. It is very difficult for many custom-made instruments to apply and measure the in-plane load. Therefore, the present MicroUTM is applied to perform the tests.

The structure of the micro-cantilever is illustrated in figure $9(a)$. The silicon cantilever is bonded onto the glass
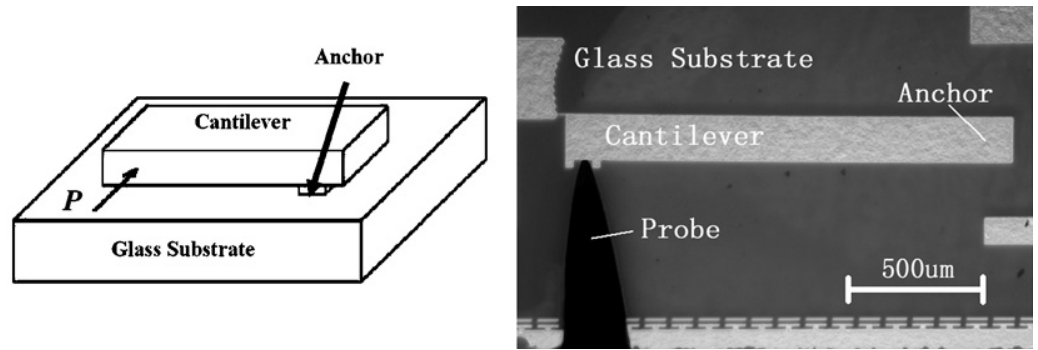

Figure 9. Illustration of the in-plane load test of the micro-cantilever using the MicroUTM: $(a)$ schematic diagram of the structure, $(b)$ a slim probe pushing the cantilever. The glass substrate of the cantilever was glued onto a piece of steel, which was mounted on the upper grip of the MicroUTM. 


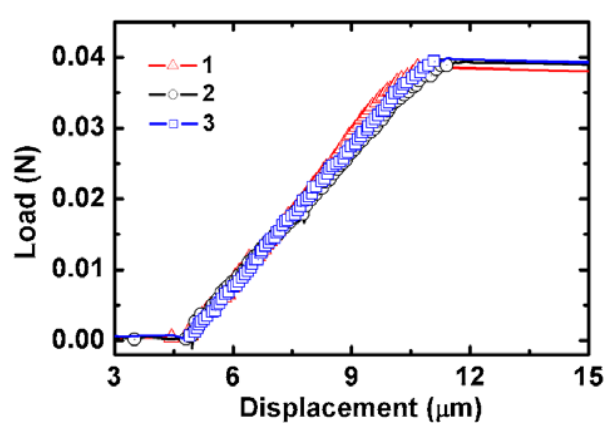

Figure 10. Load-displacement curves of three samples. Curves 1-3 represent different samples of the same size. The average ultimate load of the three samples is $38.9 \mathrm{mN} \pm 2 \%$.

substrate at the anchor. In the test, the glass substrate of these samples was glued onto a piece of steel, which was mounted on the upper grip of the present MicroUTM. A slim probe was fixed on the load axis. A linear increasing load parallel to the substrate was applied on the free end of the micro-cantilever through the probe, shown in figure $9(b)$. This process was automatically controlled and recorded by the MicroUTM.

The load-displacement curves of three samples, of the same size of $800 \mu \mathrm{m} \times 150 \mu \mathrm{m} \times 76 \mu \mathrm{m}$, are shown in figure 10. The ultimate load is $38.9 \mathrm{mN} \pm 2 \%$. Although there are several factors affecting the test results, e.g. sample preparation and test environment, the excellent repeatability of the data can still indicate that the load measurement of this tester is reliable. The typical dimension of MEMS structures that can be tested with this device is about $10^{1}-10^{3} \mu \mathrm{m}$.

\section{Summary}

In this paper, a moving-coil designed tester, named as MicroUTM, has been in-house developed for the micromechanics tension, compression and bending tests. The tester uses a moving coil suspended in a uniform magnetic field acted as the actuator as well as the load measuring means, a capacitive sensor as the displacement measuring means. The load is calibrated using the Sartorius BP211D analytical balance, with a resolution/range of $0.01 \mathrm{mg} / 80 \mathrm{~g}$ or $0.1 \mathrm{mg} / 210 \mathrm{~g}$. The displacement is calibrated using the HEIDENHAIN CT-6002 length gauge with accuracy of $\pm 0.1 \mu \mathrm{m}$. The load range is $\pm 1 \mathrm{~N}$ and the displacement range is $\pm 300 \mu \mathrm{m}$. The load nonlinearity is only $0.2 \%$, which demonstrates that the present MicroUTM can obtain a good load linearity as well as a relatively long displacement range.

Several in-plane load tests of MEMS micro-cantilevers, of size of $800 \mu \mathrm{m} \times 150 \mu \mathrm{m} \times 76 \mu \mathrm{m}$, have been successfully performed. Experimental results, with excellent repeatability, demonstrate the reliability of the load measurement as well as the flexible function of this tester.

\section{Acknowledgments}

The authors would like to thank Professor Bai Yilong for his supervision and suggestions. This work is supported by the National Natural Science Foundation of China (grant nos 10602058, 10572142 and 10432050).

\section{References}

[1] Kassner M E et al 2005 Mech. Mater. 37231

[2] Miyazaki H and Hayashi K 1999 Biomed. Microdevices 2151

[3] Menciassi A et al 2001 Microdevices 3149

[4] Sanders J E et al 2003 J. Biomed. Mater. Res. 671412

[5] Tan E P S and Lim C T 2004 Rev. Sci. Instrum. 752581

[6] Sharpe W N Jr, Yuan B and Edwards R L 1997 J. Microelectromech. Syst. 6193

[7] Tsuchiya T, Inoue A and Sakata J 2000 Sensors Actuators A 82286

[8] Augulis L et al 2004 Opt. Lasers Eng. 421

[9] Read D T et al 2001 Scr. Mater. 45583

[10] Cheng Y-W et al 2005 Thin Solid Films 484426

[11] Skove M J et al 1991 Rev. Sci. Instrum. 621010

[12] Pan Z W et al 1999 Appl. Phys. Lett. 743152

[13] Kim J, Park S and Cho D II 2002 Sensors Actuators A 97-98 653

[14] Wierzbicki R et al 2006 Microelectron. Eng. 831651

[15] Wilson C J, Ormeggi A and Narbutovskih M 1996 J. Appl. Phys. 792386

[16] Hong S H et al 2005 Compos. Sci. Tech. 651401

[17] Son D, Jeong J H and Kwon D 2003 Thin Solid Films 437182

[18] Lee H et al 2005 J. Micromech. Microeng. 151230

[19] Weihs T P, Hong S, Bravman J C and Nix W D 1988 J. Mater. Res. 3931 